\title{
Application of vibrothermography to the depth characterization of delaminations
}

\author{
by A. Salazar", A. Mendioroz , E. Apiñaniz ${ }^{*}$ A. Oleaga*, P. Venegas ${ }^{* *}$ and I. Sáez-Ocáriz ${ }^{* *}$
}

\begin{abstract}
*Departamento de Física Aplicada I, Escuela Técnica Superior de Ingeniería, Universidad del País Vasco, Alameda Urquijo s/n, 48013 Bilbao, Spain, agustin.salazar@ehu.es

${ }^{*}$ Centro de Tecnologías Aeronáuticas (CTA), Parque Tecnológico de Álava, Juan de la Cierva 1, 01510 Miñano, Spain, iocariz@ctaero.com
\end{abstract}

\begin{abstract}
In this work we present a method to evaluate the depth of delaminations in opaque samples, from lock-in vibrothermography measurements. It is theoretically demonstrated that the phase and the natural logarithm of the surface temperature above the delamination behaves linearly as a function of the square root frequency. The slope of this linear relation is directly proportional to the delamination depth. Measurements performed on composite plates with calibrated delaminations confirm the validity of the method, provided the width of the delamination is higher than the thermal diffusion length.
\end{abstract}

\section{Introduction}

Vibrothermography or thermo-sonics was introduced in the late 70 s as a new type of excitation in the field of thermographic nondestructive evaluation (NDE) for the detection of defects such as cracks or delaminations [1]. In this technique, the sample is mechanically excited by sonic or ultrasonic oscillations. This mechanical excitation acts as a selective inner heat source, located just at the defect which dissipates energy due to friction between the faces of the defect and/or stress concentration at the surrounding area. The thermal energy diffuses inside the material and can be detected as a temperature variation at its surface by means of an infrared (IR) video camera. In the case of lock-in vibrothermography [2] a high frequency oscillation is amplitude-modulated at a low frequency and the detection lock-in system (synchronized with the amplitude varying input signal) records the amplitude and phase of the surface temperature. In this work, we use lock-in vibrothermography to characterize the depth of delaminations. Measurements performed on composite plates with simulated delaminations confirm the validity of the method.

\section{Theory}

We have modelled the heat source generated at a horizontal delamination as a flat and rectangular source of length $a$ and width $b$, located at a depth $z_{0}$ beneath the surface of the sample. For the sake of simplicity, the heat source is considered homogeneous. The geometry we have worked with is shown in figure 1, where the heat source is drawn in dark grey. In lock-in vibrothermography the crack acts as a heat source modulated at the same frequency $f(\omega=2 \pi f)$ as the amplitude of the ultrasonic source. The amplitude of the temperature oscillation corresponding to the entire rectangular heat source is obtained by integrating the contributions of point-like sources covering the entire rectangular heat source:

$$
T(x, y, 0)=2 T_{o} \int_{-a / 2}^{a / 2} \int_{-b / 2}^{b / 2} \frac{e^{-q \sqrt{\left(x-x_{o}\right)^{2}+\left(y-y_{o}\right)^{2}+z_{o}{ }^{2}}}}{\sqrt{\left(x-x_{o}\right)^{2}+\left(y-y_{o}\right)^{2}+z_{o}^{2}}} d x_{o} d y_{o},
$$

where $T_{0}$ is a factor which depends on the strength of the heat source and on the thermal properties of the medium, and $q=$ $(i \omega / D)^{0.5}$ is the thermal wave vector, being $D$ the thermal diffusivity. In figure 2 we show the behaviour of the phase and of the natural logarithm of the amplitude of the surface temperature just above the delamination centre as a function of the square root frequency. As can be seen, there is a linear relation whose slope is given by: $m=-z_{0}(\pi / D)^{0.5}$. Accordingly, the delamination depth can be obtained if the thermal diffusivity of the sample is known. This linear relation holds provided the two following conditions are fulfilled: (a) the thermal diffusion length, $\mu=(D / \pi f)^{0.5}$, is higher than the delamination depth and (b) the thermal diffusion length is smaller than the delamination size ( $a$ and $b$ ). 


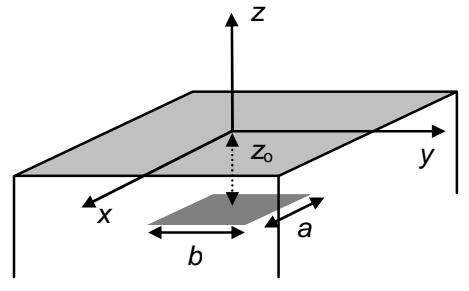

Fig. 1. Geometry of the problem. The sample surface is represented in light grey, while the subsurface delamination in dark grey.

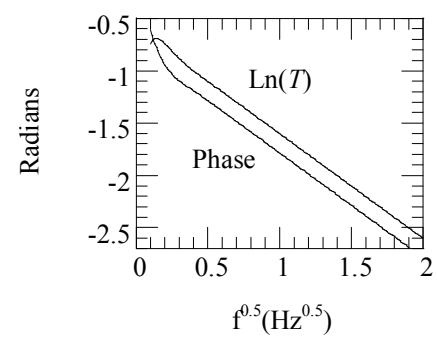

Fig. 2. Phase and natural logarithm of the surface temperature as a function of $f^{0.5} \cdot a=b=10 \mathrm{~mm}$, $z_{0}=-0.4 \mathrm{~mm}, D=0.5 \mathrm{~mm}^{2} / \mathrm{s}, K=2 \mathrm{Wm}^{-1} \mathrm{~K}^{1}$.

\section{Experimental results and discussion}

To verify the validity of the method we have prepared calibrated delaminations by inserting four squared films of Teflon between two plies of a CFRC plate $1 \mathrm{~cm}$ thick. The size of the films simulating the delamination is $15 \mathrm{~mm} \times 15 \mathrm{~mm}$, with increasing depths: (a) $0.5 \mathrm{~mm}$, (b) $1 \mathrm{~mm}$, (c) $1.5 \mathrm{~mm}$ and (d) $2.0 \mathrm{~mm}$. The measurements were performed with a commercial lock-in vibrothermography equipment (UTvis from EDEVIS). The surface temperature data were collected with an infrared camera (Silver 480M from CEDIP), synchronized with the amplitude varying input signal.

Figure 3 shows the phase thermogram of the CFRC plate at two modulation frequencies: 0.1 and $1 \mathrm{~Hz}$. The simulated delaminations are clearly detected although the deepest one is close to the detection limit. As a further step, we are interested in measuring their depths. To do this we performed several measurements varying the modulation frequency in the range from $0.01 \mathrm{~Hz}$ to $5 \mathrm{~Hz}$. The phase of the surface temperature at the centre of the delamination is depicted as a function of the square root frequency. The results for the delaminations labelled as (a), (b) and (c) in figure 3 are shown in figure 4. From the slope of each straight line the delamination depth was obtained taking the thermal diffusivity of the CFRC as $D=0.45 \mathrm{~mm}^{2} / \mathrm{s}$. The obtained depths are $0.45 \pm 0.05 \mathrm{~mm}, 1.11 \pm 0.15 \mathrm{~mm}$, and $1.3 \pm 0.3 \mathrm{~mm}$ respectively. The agreement with the actual depths is very good, but the uncertainty increases with the depth of the delamination. The data for the deepest one, labelled as (d), are not good enough to retrieve its depth. Similar results have been obtained from the amplitude values, but the uncertainty is greater.

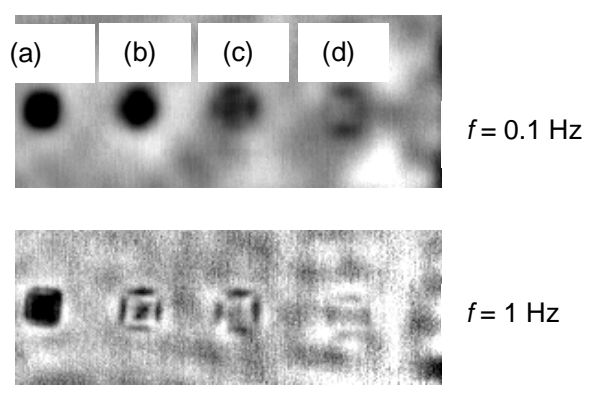

Fig. 3. Phase thermograms of the CFRC plate with four buried delaminations. Their depths are: (a) $z_{0}=-0.5 \mathrm{~mm}$, (b) $z_{0}=-1.0 \mathrm{~mm}$, (c) $z_{0}=-1.5$ $\mathrm{mm}$ and $(d) z_{o}=-2.0 \mathrm{~mm}$.

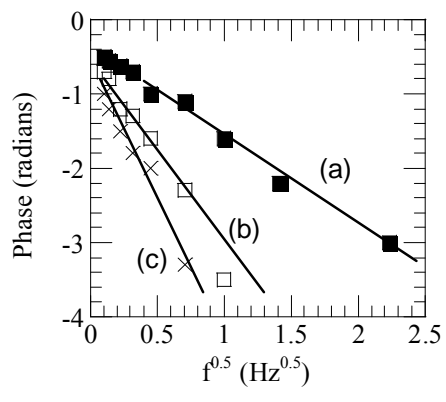

Fig. 4. Experimental evolution of the phase of the surface temperature as a function of $f^{0.5}$ for the delaminations (a), (b) and (c) in figure 3. Continuous lines are the linear fits of the data.

This work has been supported by the Ministerio de Educación y Ciencia (MAT2008-01454) and by The University of the Basque Country (DIPE08/10).

\section{REFERENCES}

[1] Carlomagno G.M. and Berardi P.G., Proc. $3^{\text {rd }}$ Biannual Exchange 33,1976.

[2] Rantala J., Wu D. and Busse G., Res. Nondestr. Eval. 7, p. 215,1996. 